\title{
Neurite Outgrowth Inhibitors in Gliotic Tissue
}

\author{
M. Nieto-Sampedro, P. Bovolenta and F. Wandosell \\ Neural Plasticity Group, \\ Instituto Cajal, C.S.I.C., Madrid, Spain
}

\begin{abstract}
Reactive gliosis is a general response of mammalian central nervous system (CNS) to injury. Traditionally, the glial scar has been viewed as the major obstacle to axon regeneration (and hence, to host-transplant integration), but a direct test of this idea has been hampered by the lack of an in vitro model of reactive glia. Cultured perinatal astrocytes, treated with dibutyryl-cyclic-AMP (dBcAMP), had a morphology and intermediate filament content reminiscent of reactive astrocytes evoked by injury. However, their immunohistochemical and neurite outgrowth-promoting properties were much closer to those untreated astrocytes in culture than to reactive astrocytes in vivo (Bovolenta P, Wandosell F, Nieto-Sampedro M, Res Neurol Neurosci 1991; 2: 221-228). Our observations strongly suggest that diBcAMP treatment leads, at best, to a morphological model of reactive cells, lacking many properties of reactive astrocytes generated in vivo. Accordingly, we developed an alternative model, based on the fact that plasma membranes can be easily purified, so that they conserve many of the surface properties of the cell. Myelin-free plasma membranes, purified from normal and gliotic tissue by a combination of differential and sucrose gradient centrifugation, were used as cell culture substrate for i) neurite initiation and ii) neurite choice of growth substrate.

To test neurite initiation, purified plasma membranes alone or mixed with laminin (LN) were adsorbed onto polylysine (PLL)-coated tissue culture dishes. Neurite outgrowth from rat embryo explants or dissociated cells (septum, hippocampus, or dorsal root ganglia), was determined conventionally, using as controls purified myelin, that strongly inhibited neurite outgrowth, and membranes from cultured type 1
\end{abstract}

astrocytes, that were powerful neurite growth promoters. For the substrate choice experiments, neurite outgrowth was initiated by excess PLL-LN and the growing neurites were faced with a substrate coated with PLL-LN and the test substance. The behavior of the growth cone, when reaching the boundary between the two substrates, was recorded.

Normal brain tissue membranes (NTM) promoted neurite initiation and provided a permissive substrate for neurite extension. In contrast, membranes purified from isomorphic gliotic tissue inhibited neurite initiation and repelled growing neurites. The inhibitory activity was solubilized using a mild detergent and was subsequently purified and characterized using conventional biochemical techniques, under denaturing conditions. The activity resided in a proteoglycan fraction of molecular weight 150$200 \mathrm{kDa}$ that was heat, trypsin, lipase and glycosidase resistant, but glycosaminoglycan lyasesensitive. Digestion with a combination of heparinase I and chondroitinase $A B C$ revealed core proteins of apparent molecular weight 66$68 \mathrm{kDa}$ and $48 \mathrm{kDa}$. The expression of the 48 $\mathrm{kDa}$ protein underwent a large specific increase in injured vs normal brain tissue. The inhibitory proteoglycan was structurally and immunologically different from the myelin inhibitors. When presented as a surface bound molecule it prevented neurite initiation. When added in a soluble form to already growing neurites, it induced their retraction. Purified microglial membranes contained neurite inhibitory activity with properties similar to those of the proteoglycan purified from gliotic tissue. Furthermore, a polyclonal antibody to the inhibitory fraction appeared to immunostain microglial cells in culture. CNS tissue, it appears, 
always contains both neurite-promoting and neurite-inhibiting molecules. It is the balance of these two activities that determines how good an environment for neurite outgrowth is provided by the CNS under given physiological conditions. Our results provide the first cellular and molecular evidence in support of the classical view that, in the mammalian CNS, injury evoked gliosis enhances the expression of molecules capable of preventing nerve regeneration.

Supported by Grant FAR 89-0683 from the National Program for Pharmaceutical Research. PB was supported by a postdoctoral fellowship from the Department of Education and Science. 

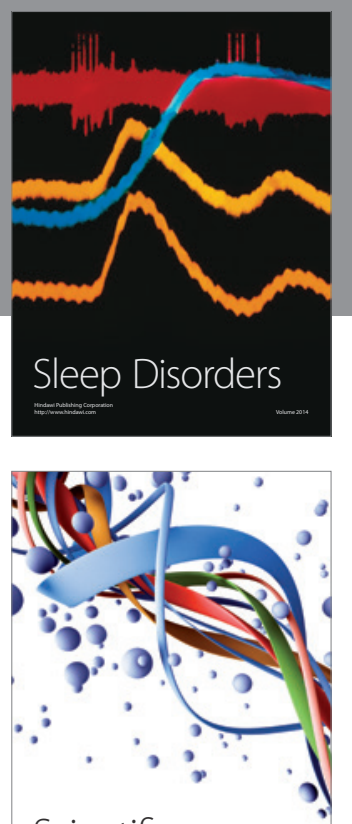

Scientifica
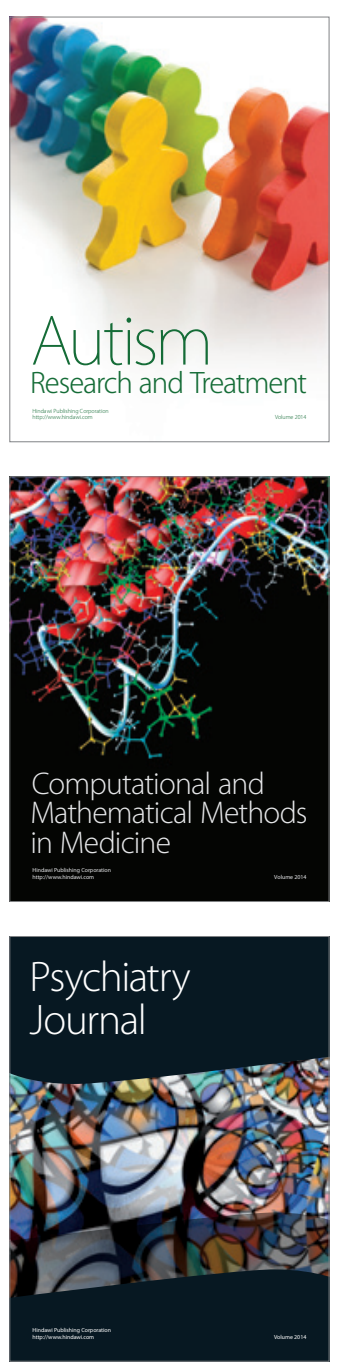
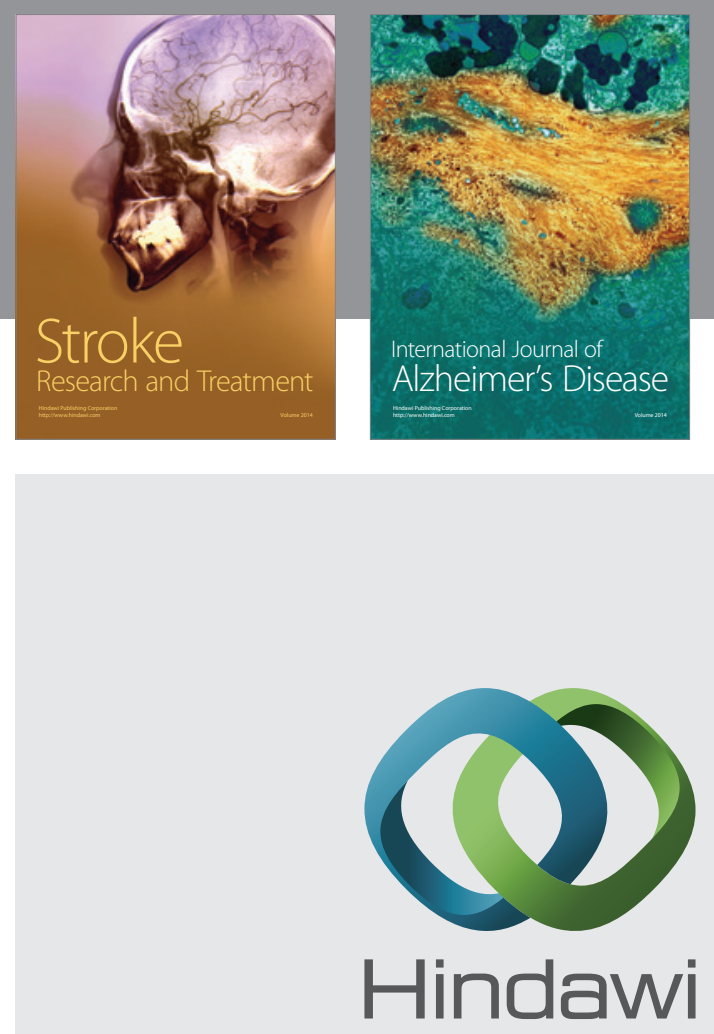

Submit your manuscripts at

http://www.hindawi.com
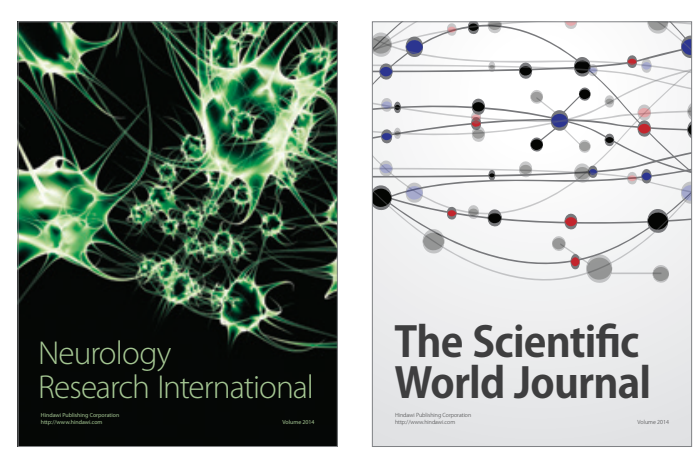

The Scientific World Journal

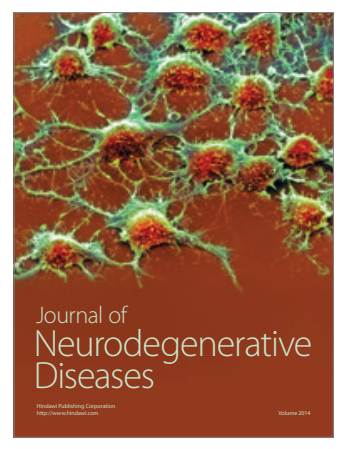

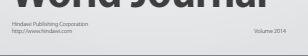

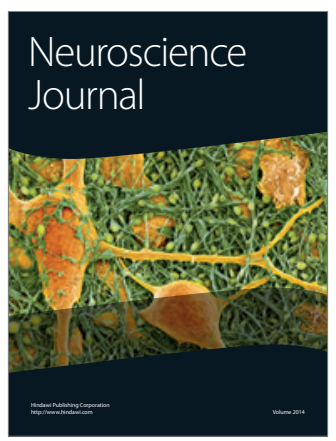

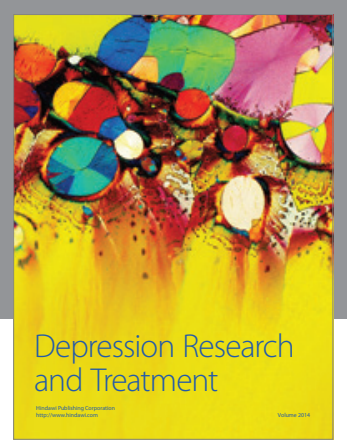
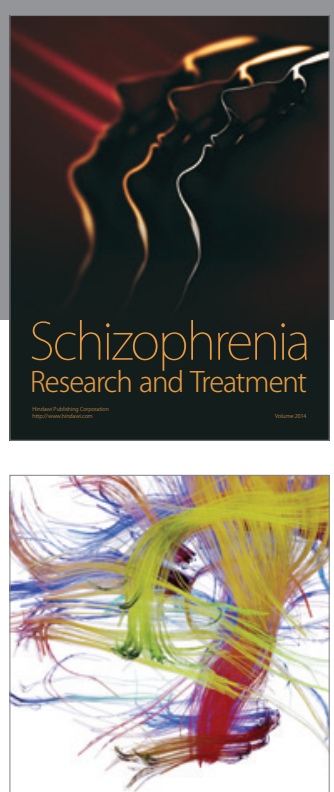

Brain Science

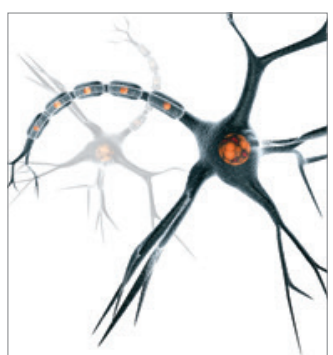

Neural Plasticity
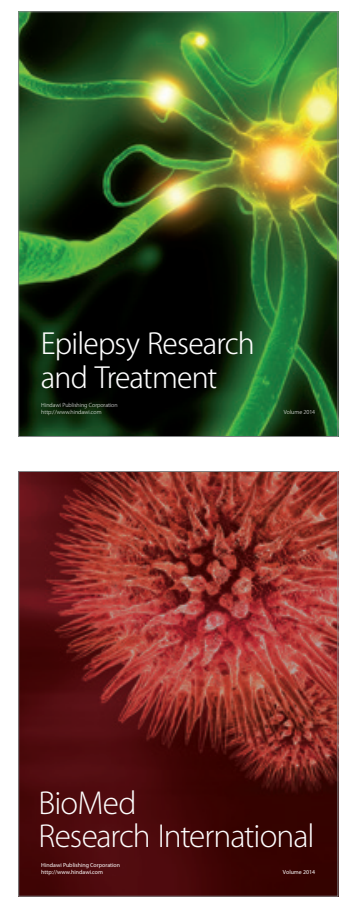

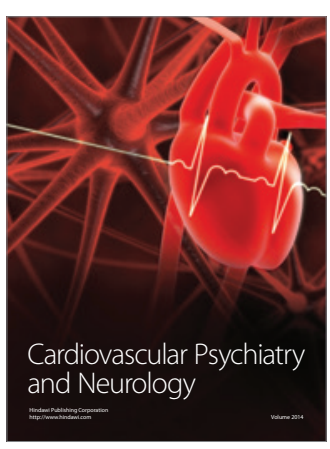

Parkinson's

Disease
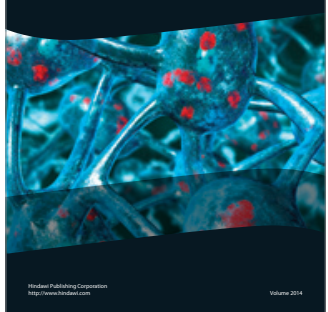